\title{
Quantum Metrology Assisted by Abstention
}

\author{
B. Gendra, E. Ronco-Bonvehi, J. Calsamiglia, R. Muñoz-Tapia, and E. Bagan \\ Física Teòrica: Informació i Fenòmens Quàntics, Departament de Física, Universitat Autònoma de Barcelona, \\ 08193 Bellaterra (Barcelona), Spain
}

(Received 4 October 2012; published 4 March 2013)

\begin{abstract}
The main goal of quantum metrology is to obtain accurate values of physical parameters using quantum probes. In this context, we show that abstention, i.e., the possibility of getting an inconclusive answer at readout, can drastically improve the measurement precision and even lead to a change in its asymptotic behavior, from the shot-noise to the Heisenberg scaling. We focus on phase estimation and quantify the required amount of abstention for a given precision. We also develop analytical tools to obtain the asymptotic behavior of the precision and required rate of abstention for arbitrary pure states.
\end{abstract}

PACS numbers: 03.67. $-\mathrm{a}, 03.65 . \mathrm{Ta}, 06.20 .-\mathrm{f}$

Precision measurements play a key role in all sciences and branches of technology. In this framework, quantum systems have long been recognized to provide a substantial precision enhancement in some important cases which, along with recent advances in the control of individual quantum systems, has fired broad interest in quantum metrology [1]. In its simplest form, a quantum metrology problem has the following structure. A quantum system undergoes some physical interaction determined by some continuous parameters. The value of the parameters gets imprinted onto the evolved state and the task of the metrologist consists in uncovering it. For this purpose, she performs a suitable measurement on the system and, based on its outcome, she produces a guess for the unknown value of the parameters as accurate as possible. The overall performance of the whole procedure can be quantified by the average of a figure of merit (typically the fidelity) over some $a$ priori distribution of the parameters and over all possible outcomes. Phase estimation with pure states of $N$ qubits is a paradigmatic example of this endeavor and will serve as the main exemplification of our findings.

In standard parameter estimation protocols [2-5] the experimentalist is expected to produce a conclusive answer (maybe not right or accurate enough), at each run of the experiment. Here we show that there are situations where the ultimate precision of the standard approach can be improved substantially if one allows for a number of inconclusive responses, where the metrologist abstains from producing a guess. This is especially relevant in situations where she can afford to rerun the experiment (i.e., she can easily prepare a new instance of the problem) or where she prioritises having high-quality estimates.

Abstention has already been used in the context of state discrimination [6-9], where some fixed rate $Q$ of inconclusive outcomes can lower the probability of error significantly (even down to zero, as in unambiguous discrimination [6]), and can be seen as a particular example of postselection [10]. In the estimation framework the effects of abstention have hardly been considered: Reference [11] is concerned with computing the maximum estimation improvement with unbounded abstention (which renders the procedure unsuitable for many practical purposes), while in [12] a fixed amount of abstention is considered, though the analysis is restricted to uncorrelated probes. In both cases abstention has limited impact for large samples unless arbitrarily large abstention is allowed.

In this Letter we show that when quantum correlations are taken into account, a limited amount of abstention can have a dramatic effect, in some cases leading to the Heisenberg instead of the shot-noise scaling. Though we focus on phase estimation, other problems, such as direction estimation, can be tackled in the same way (results will be given elsewhere [13]). We also present a very general technique to obtain the asymptotic form of pure-state parameter estimation problems, with or without abstention. It is worth mentioning that even in the standard approach to estimation (without abstention) analytical asymptotic expressions were known in just a few cases.

In phase estimation, one aims to optimally estimate the parameter $\theta$ of the covariant family of states $\{|\Psi(\theta)\rangle=$ $\left.U(\theta)\left|\Psi_{0}\right\rangle\right\}_{\theta \in[0,2 \pi)}$, where $U(\theta)$ stands for the unitary transformation $U(\theta)|j\rangle=e^{i \theta j}|j\rangle$, and $\left|\Psi_{0}\right\rangle$ is a normalized fiducial state that in the eigenbasis of $U(\theta)$ can be written as $\left|\Psi_{0}\right\rangle=\sum_{j=0}^{N} c_{j}|j\rangle \in\left(\mathbb{C}^{2}\right)^{\otimes N}$. The fidelity between the true phase $\theta$ and its estimate $\theta_{\chi}$ can be written as $[1+$ $\left.\cos \left(\theta-\theta_{\chi}\right)\right] / 2$, where $\chi$ is a subscript specifying that the estimate is based on the outcome $\chi$ of a generalized measurement. This is characterized mathematically by a positive operator valued measure (POVM) $\Pi=\left\{\Pi_{\chi}\right\} \cup$ $\left\{\Pi_{0}\right\}, \Pi_{0}+\Sigma_{\chi} \Pi_{\chi}=\mathbb{1}$, where $\Pi_{\chi} \geq 0$ outputs a conclusive answer (from which an estimate is proposed) and $\Pi_{0} \geq 0$ outputs "abstention." The probability of abstention taking place is

$$
Q=\int \frac{d \theta}{2 \pi}\left\langle\Psi(\theta)\left|\Pi_{0}\right| \Psi(\theta)\right\rangle
$$


and $\bar{Q}=1-Q$ is the acceptance probability (rate at which we provide definite estimates). The average fidelity for this rate of abstention is

$F(Q)=\frac{1}{\bar{Q}} \sum_{\chi} \int \frac{d \theta}{2 \pi} \frac{1+\cos \left(\theta-\theta_{\chi}\right)}{2}\left\langle\Psi(\theta)\left|\Pi_{\chi}\right| \Psi(\theta)\right\rangle$.

Estimation with abstention can be reduced to a standard estimation problem by simply introducing the new POVM $\tilde{\Pi}$, with elements given by $\tilde{\Pi}_{\chi} \equiv\left(\mathbb{1}-\Pi_{0}\right)^{-1 / 2} \Pi_{\chi}$ $\left(\mathbb{1}-\Pi_{0}\right)^{-1 / 2}$, and the new family of (normalized) states

$$
\left\{|\tilde{\Psi}(\theta)\rangle \equiv \frac{\left(\mathbb{1}-\Pi_{0}\right)^{1 / 2}}{\bar{Q}^{1 / 2}}|\Psi(\theta)\rangle\right\}_{\theta \in[0,2 \pi)} .
$$

This formulation brings forward an interpretation of the role of abstention here: each initial state $|\Psi(\theta)\rangle$ is transformed into a new $|\tilde{\Psi}(\theta)\rangle$ that "encodes" the unknown parameter $\theta$ in a more efficient way. This map improves the estimation precision by effectively increasing the distinguishability between the signal states; therefore, it can only be implemented in a probabilistic fashion (it succeeds with probability $\bar{Q}$ ). This stochastic map is fully specified by the optimal choice of $\Pi_{0}$, i.e., the one that maximizes (2). Although this may seem a difficult optimization problem, a huge simplification arises because of the covariance of the family of states. Already from Eqs. (1) and (2) one can easily see that the optimal POVM can be chosen to be covariant under the set of unitaries $\{U(\theta)\}$. In particular this means that $\Pi_{0}$ can be taken invariant under the transformations $\{U(\theta)\}$. Thus, using Shur's lemma one gets $\Pi_{0}=\sum_{j} f_{j}|j\rangle\langle j|$, and the maximization is over $\left\{f_{j}: 0 \leq f_{j} \leq 1\right\}_{j=0}^{N}$. Note that the transformed set of states $\{|\tilde{\Psi}(\theta)\rangle\}$ is also a covariant family, just as the original one, with fiducial state

$$
\left|\tilde{\Psi}_{0}\right\rangle=\sum_{j=0}^{N} \frac{c_{j} \sqrt{\bar{f}_{j}}}{\sqrt{\bar{Q}}}|j\rangle=\sum_{j=0}^{N} \xi_{j}|j\rangle \equiv|\xi\rangle,
$$

where $\bar{f}_{j} \equiv 1-f_{j}$.

Since the transformed states are still covariant, we can choose $\tilde{\Pi}$ to be the well-known optimal continuous and covariant POVM [2,5]: $\quad\{\tilde{\Pi}(\theta)=U(\theta)|\Phi\rangle \times$ $\left.\langle\Phi| U^{\dagger}(\theta)\right\}_{\theta \in[0,2 \pi)}$, where $|\Phi\rangle=\sum_{j=0}^{N}|j\rangle$. Hereafter it is assumed that the states have non-negative coefficients $\xi_{j} \geq 0$ (and hence $c_{j} \geq 0$ ), because any phases present in the coefficients $c_{j}$ (or $\xi_{j}$ ) can be absorbed by the above POVM elements. With this, the calculation of the fidelity simplifies to

$$
F=\frac{1}{2}+\frac{1}{2}\langle\xi|\mathrm{M}| \xi\rangle,
$$

where in the eigenbasis of $U(\theta)$ the matrix of $\mathrm{M}$ is real and tridiagonal, with elements $M_{i j}=\left(\delta_{i, j+1}+\delta_{i, j-1}\right) / 2$. The maximization over $\left\{f_{j}\right\}$ in (5) can be turned into a maximization over the transformed states, $\Delta \equiv \max _{|\xi\rangle}\langle\xi|\mathrm{M}| \xi\rangle=\max _{\left\{\xi_{j}\right\}} \sum_{j=0}^{N-1} \xi_{j} \xi_{j+1}$, subject to the constraints $\langle\xi \mid \xi\rangle=1$ and $\xi_{j} \leq \lambda c_{j}$, where $\lambda \equiv \bar{Q}^{-1 / 2}$. Then, the maximum fidelity for a given rate of abstention $Q$ is $F(Q)=(1+\Delta) / 2$.

For large enough abstention rates the inequality constraint has no effect (provided $c_{j} \neq 0, \forall j$ ) and $\Delta$ becomes the maximum eigenvalue of the matrix $\mathrm{M}$. In this case, $F^{*} \equiv$ $F(Q \rightarrow 1)$ is the maximum fidelity that can be achieved by optimizing the components of the fiducial state; these are given by the corresponding eigenvector $\left|\xi^{*}\right\rangle$ of $\mathrm{M}$ [11]. From the inequality constraint we obtain the critical acceptance rate $\bar{Q}^{*}=\min _{j}\left(c_{j} / \xi_{j}^{*}\right)^{2}$. That is, for abstention rates such that $Q \geq Q^{*}=1-\bar{Q}^{*}$ the fidelity attains its absolute maximum value $F^{*}$. In the other extreme, when no abstention is allowed $(Q=0)$, no maximization is possible; hence, $\xi_{j}=c_{j}$ and $\Delta=\langle c|\mathrm{M}| c\rangle$. For intermediate values of $Q \in$ $\left(0, Q^{*}\right)$ the calculation becomes more tricky. For moderate values of $N$, it can be easily cast as a semidefinite programming (SDP) problem (see Supplemental Material [14]) and hence solved efficiently to arbitrary accuracy [15].

However, the main focus of this work is on asymptotics (large- $N$ regime) and, in particular, on presenting an approach that enables obtaining analytical asymptotic expressions. With this aim, let us define $S \equiv 1-\langle\xi|\mathrm{M}| \xi\rangle$, where we further introduce Lagrange multipliers $b^{2} / 2$ for the normalization condition and $s_{j}, j=0, \ldots, N$ for the inequality constraints $\xi_{j} \leq \lambda c_{j}$, also called primal feasibility conditions. We thus have to minimize

$$
\begin{aligned}
S= & \frac{1}{2}\left[\sum_{j=0}^{N-1}\left(\xi_{j+1}-\xi_{j}\right)^{2}+\xi_{1}^{2}+\xi_{N}^{2}\right] \\
& -\frac{b^{2}}{2}\left(\sum_{j=0}^{N} \xi_{j}^{2}-1\right)+\sum_{j=0}^{N} s_{j}\left(\xi_{j}-\lambda c_{j}\right),
\end{aligned}
$$

where the so-called dual feasibility conditions $s_{j} \geq 0$, and the complementary slackness conditions $s_{j}\left(\xi_{j}-\lambda c_{j}\right)=0$ must also be imposed, as dictated by the Karush-KuhnTucker (KKT) method (see, e.g., [15]).

Rather than attempting to solve the above minimization, we will reframe it as a functional variational problem by taking $N$ to be asymptotically large. We first note that as $N$ goes to infinity, $j / N$ approaches a continuous real variable $t$. So, we define $0 \leq t \equiv j / N \leq 1$, and assume $\left\{\xi_{j}\right\}$ and $\left\{c_{j}\right\}$ are a discretization of some continuous functions, $\varphi(t)=\xi_{j} \sqrt{N}$ and $\psi(t)=c_{j} \sqrt{N}$. Note that $\varphi(t) \geq 0$ and $\psi(t) \geq 0$, and the normalization condition $\int_{0}^{1} \varphi(t)^{2}=$ $\int_{0}^{1} \psi(t)^{2}=1$ is satisfied. It follows that $\xi_{j+1}-\xi_{j} \simeq$ $N^{-3 / 2}[d \varphi(t) / d t]$, and we can write (6) as the functional

$$
\begin{gathered}
S[\varphi]=\frac{\varphi^{2}(0)+\varphi^{2}(1)}{2 N}+\frac{1}{N^{2}} \int_{0}^{1} d t\left[\frac{1}{2}\left(\frac{d \varphi}{d t}\right)^{2}\right. \\
\left.-\frac{\omega^{2}}{2}\left(\varphi^{2}-1\right)+\sigma(\varphi-\lambda \psi)\right],
\end{gathered}
$$


where $\omega=N b \geq 0$ is the properly scaled Lagrange multiplier and $\sigma(t)$ is a function that interpolates the set of multipliers $\left\{s_{j}\right\}$, i.e., $s_{j}=N^{-5 / 2} \sigma(t)$. With this, the following conditions must hold: $\varphi(t)-\lambda \psi(t) \leq 0$ (primal feasibility), $\sigma(t) \geq 0$ (dual feasibility), and $\sigma(t) \times$ $[\varphi(t)-\lambda \psi(t)]=0$ (complementary slackness).

Note that by imposing the boundary conditions $\varphi(0)=$ 0 and $\varphi(1)=0$, the first (shot-noise scaling) term of $O\left(N^{-1}\right)$ is cancelled. These conditions can be always met if $Q>0$. Furthermore, if $\psi(t)$ has wide (finite) supporti.e., $c_{j}>0$ over a large (order $N$ ) range of $j$-the integral in (7) will be finite as $N \rightarrow \infty$ and the functional $S[\varphi]$ will become $O\left(N^{-2}\right)$; i.e., Heisenberg scaling will be attained.

The minimization of $S[\varphi]$ defines a mechanical problem, of which the second line in Eq. (7) is the action and the corresponding integrand the Lagrangian. It describes a driven harmonic oscillator with angular frequency $\omega$, whose equation of motion is

$$
\frac{d^{2} \varphi}{d t^{2}}+\omega^{2} \varphi=\sigma
$$

To solve this problem, we first note that the slackness conditions imply that either $\varphi(t)=\lambda \psi(t)$, in which case we say $t$ is in the so-called coincidence set $\mathcal{C}$, or $\sigma(t)=0$. In the second case, $t \in \overline{\mathcal{C}}$ (the complement of $\mathcal{C}$ ), the primal feasibility condition is $\varphi(t)<\lambda \psi(t)$ and Eq. (8) becomes homogeneous. It has the familiar solution $\varphi(t)=$ $A \sin \omega t+B \cos \omega t$, where $A, B$, and $\omega$ are constants to be determined. In $\mathcal{C}, \sigma$ is given by (8), where we make the substitution $\varphi(t)=\lambda \psi(t)$. If we restrict ourselves to fiducial states $\left|\Psi_{0}\right\rangle$ whose components $c_{j}$ are such that $\psi(t)$ is continuous in the whole unit interval, one can show that the solution $\varphi(t)$ and its first derivative must be also continuous there [except in points of $\mathcal{C}$ where $\psi(t)$ itself is not differentiable]. Most of the physically relevant cases are of this type. By taking into account the boundary conditions, as well as the "matching conditions," namely, continuity of $\varphi(t)$ and its derivative in the boundaries of $\mathcal{C}$, one can determine the arbitrary constants that arise in solving the equation of motion, which include the location of the boundaries of $\mathcal{C}$. The minimum value of $S$ for $Q>0$ can be expressed in terms of the Lagrange multiplier (function) $\omega(\sigma)$, and the given function $\psi$, as

$$
S=\frac{1}{N^{2}}\left(\frac{\omega^{2}}{2}-\frac{\lambda}{2} \int_{0}^{1} d t \sigma \psi\right) .
$$

Note that the integral is effectively over $\mathcal{C}$, where $\sigma=$ $\lambda\left(d^{2} \psi / d t^{2}+\omega^{2} \psi\right)$. In the following we solve some relevant cases, but we first compute the fidelity for arbitrary large rates of abstention, assuming $c_{j} \neq 0, \forall j$.

Large abstention.-For abstention rates very close to one (large $\lambda$ ), one has $\mathcal{C}=\varnothing$, and the above reduces to a maximum eigenvalue problem. The solution is $\varphi(t)=$ $\sqrt{2} \sin \pi t$. This yields the asymptotic result

$$
F^{*}=1-\frac{\pi^{2}}{4 N^{2}},
$$

which coincides with the known maximum fidelity for optimal phase encoding [11,16].

Phase states.-The elements of this family are generated by an equal superposition of all Fock states $|j\rangle, c_{j}=$ $1 / \sqrt{N+1}$ (hence proportional to the POVM seed state). The corresponding continuous version is $\psi(t)=1$. Without abstention, $Q=0(\lambda=1)$, the optimal phase estimation precision provided by these states does not exceed the shot-noise limit: $1-F=1 /(2 N+2)$. For $Q>0(\lambda>1)$ the situation changes markedly. We can freely impose $\varphi(0)=\varphi(1)=0$ and get rid of the shotnoise type of term $1 / N$ in (7). In a sufficiently small neighborhood of $t=0$, we have $\varphi(t)-\lambda<0$, and hence $\sigma(t)=0$ there. If $\alpha$ is the maximum value of $t$ less than $1 / 2$ for which this condition holds, it must be a boundary point of $\mathcal{C}$. Then, for $\alpha \leq t \leq 1 / 2$ the solution is given by the rescaled input state $\varphi(t)=\lambda \psi(t)=\lambda$. Thus, $\varphi(t)=$ $A \sin \omega t$ for $t \in[0, \alpha)$ and $\varphi(t)=\lambda$ for $t \in[\alpha, 1 / 2]$. Imposing the matching conditions we find $\alpha=1-1 / \lambda^{2}=$ $Q, \lambda \leq \sqrt{2}$ (i.e., $Q^{*}=1 / 2$ ), and

$$
\varphi(t)= \begin{cases}\bar{Q}^{-(1 / 2)} \sin \frac{\pi t}{2 Q} & 0 \leq t<Q, \\ \bar{Q}^{-(1 / 2)} & Q \leq t \leq 1 / 2 .\end{cases}
$$

For $1 / 2<t \leq 1$ the solution follows from the obvious symmetry relation $\varphi(t)=\varphi(1-t)$. We have $\mathcal{C}=[Q, \bar{Q}]$ and $\sigma(t)=\omega^{2} \lambda$ for $t \in \mathcal{C}$. Therefore, Eq. (9) leads to

$$
F=1-\frac{\pi^{2}}{16 Q \bar{Q} N^{2}}, \quad 0<Q \leq Q^{*}=1 / 2,
$$

and for $1 / 2<Q \leq 1$ we have $F=F^{*}$ [see Eq. (10)]. We note that even the slightest abstention rate unlocks the probing capabilities of the phase states and drastically changes the estimation precision from shot-noise $(1 / N)$ to Heisenberg $\left(1 / N^{2}\right)$ scaling [17].

Multiple copies.-These probe states have been widely considered in quantum metrology. They have the form

$$
|\Psi(\theta)\rangle=\left(\frac{|0\rangle+e^{i \theta}|1\rangle}{\sqrt{2}}\right)^{\otimes N}
$$

and the coefficients of the corresponding fiducial state $\left|\Psi_{0}\right\rangle$ read $c_{j}=2^{-N / 2}\left({ }_{j}^{N}\right)^{-1 / 2}$. Their maximum precision for $Q=0$ and large $N$ is known to be $1-F=1 /(4 N)$ [16].

In the asymptotic limit the rescaled components $\sqrt{N} c_{j}$ approach the function

$$
\psi(t)=\left[\frac{N}{2 \pi t(1-t)}\right]^{1 / 4} \exp \left\{-\frac{N}{2} H(t \| 1 / 2)\right\},
$$

where $H(t \| 1 / 2)=\log 2+t \log t+(1-t) \log (1-t)$ is the relative entropy between two Bernoulli distributions with success probabilities $t$ and $1 / 2$. The symmetry of 

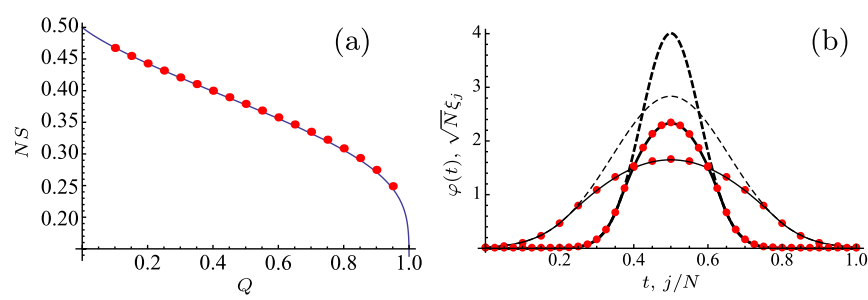

FIG. 1 (color online). (a) Plot of $N S=2 N(1-F)$ vs $Q$ (solid line) for an asymptotically large number $N$ of parallel spins, Eq. (13). The dots have been obtained by numerical optimization with $N=100$. (b) Profile of the transformed fiducial state $\left|\tilde{\Psi}_{0}\right\rangle$ for $\lambda=1.5(Q=0.56)$. The thin (thick) line corresponds to $N=20(N=80)$. The points are obtained by numerical optimization. The dashed lines represent the function $\lambda \psi(t)$ that defines the primal feasibility condition $\varphi(t) \leq \lambda \psi(t)$, where $\psi(t)$ is in (14).

the problem suggests using the variable $\tau=t-1 / 2$, $\tau \in[-1 / 2,1 / 2]$. As a function of this new variable $\varphi(\tau)$ must be even [we slightly abuse notation by writing $\varphi(t(\tau))$ in short as $\varphi(\tau)$ ]; hence, it must have the form

$$
\varphi(\tau)= \begin{cases}A \cos \omega \tau, & 0 \leq|\tau| \leq \alpha \\ \lambda \psi(\tau), & \alpha<|\tau| \leq 1 / 2\end{cases}
$$

where as above $\omega, \alpha$ and $A$ are determined by the matching and normalization conditions. The resulting equations cannot be solved in full generality; however, we will obtain analytical solutions in two relevant regimes. To this end, we note that in the region $|\tau| \lesssim N^{-1 / 2}$ [i.e., around the peak at $t=1 / 2$ of (14)], $\psi(\tau)$ behaves as the Gaussian distribution $\psi(\tau) \approx(2 N / \pi)^{1 / 4} e^{-N \tau^{2}}$, whereas at the tails $\left(|\tau|>N^{-1 / 2}\right)$ it falls off at an exponential rate lower bounded by $H(1 / 2+\alpha \| 1 / 2) / 2$.

In the first regime, the abstention rate $Q$ has a fixed nonzero value (independent of $N$ ). This requires that the boundary point $\alpha$ scales as the width of $\psi(\tau)$, i.e., $\alpha \sim N^{-1 / 2}$; thus $\psi(\tau)$ is accurately given by its Gaussian approximation. This observation, along with a suitable rescaling of $\omega$ and $A$, enables us to obtain the solution in parametric form: $(Q(\Omega), S(\Omega)$ ) (see Supplemental Material [14]). In Fig. 1(a) we plot $N S=2 N(1-F)$ as a function of $Q$. Despite the strong dependence on the abstention rate, particularly at $Q \lesssim 1$, the precision will be shot-noise limited in the whole interval $[0,1)$. We see that, e.g., an abstention of about $90 \%$ has the same effect as doubling the number of copies in the standard approach, with $Q=0$. Also, for vanishing abstention rate we recover the well-known result $2 N(1-F)=1 / 2$. The exact (numerical) profile of the transformed state $\left|\tilde{\Psi}_{0}\right\rangle$ is shown in Fig. 1(b), together with the analytical result $\varphi(t)$, for an abstention rate of $56 \%$ and two different values of 20 and 80 copies for $N$.

We next explore a second regime in which the Heisenberg limit $1-F \sim N^{-2}$ (i.e., $S \sim N^{-2}$ ) is attained. For that we need that the function $\varphi(\tau)$ in (15) has a wide (nonvanishing) support as $N$ goes to infinity. This is easily accomplished by taking the boundary point $\alpha$ to be independent of $N$. In this situation, once $N$ is large enough, the coincidence set $\mathcal{C}$ lies on the tails of $\psi(\tau)$, where this function falls off exponentially at the dominant rate of $H(1 / 2+\alpha \| 1 / 2) / 2$. Solving the matching and normalization conditions, we obtain the fidelity,

$$
F=1-\frac{\pi^{2}}{16 N^{2} \alpha^{2}}+O\left(N^{-3}\right) ; \quad 0<\alpha \leq 1 / 2
$$

and the acceptance rate, $Q \sim \exp \{-N H(1 / 2+\alpha \| 1 / 2)\}$. Thus, the Heisenberg limit scaling $1 / N^{2}$ can be attained if such an exponential rate of acceptance is affordable. Note that the critical acceptance rate is $\bar{Q}^{*}=2^{-N}(\alpha \rightarrow 1 / 2)$, below which $F=F^{*}$.

Although in this Letter we have focused on phase estimation, our methods directly apply to other problems such as direction estimation [13]. In this case one can define the analogous of the two fiducial states introduced above. Our results nicely mirror those presented here, the main difference being the replacement of the trigonometric functions by Bessel functions.

Recent works [18] show that under quite general conditions, noise renders (asymptotic) Heisenberg scaling unattainable. A quantitative study of the benefits of abstention in general noisy scenarios requires to extend the presented methods to mixed states [13]. Following similar arguments as in [18] and considering some particular noise sources, one can check that abstention will not be able to restore the Heisenberg scaling. However, abstention can still provide a significant enhancement in precision, especially for moderate (experimentally relevant) values of $N$; see also [12]. The role of abstention with restricted measurement capabilities, such as local addressing or other experimentally motivated constraints, is also a relevant open question that requires further study.

We thank G. Chiribella for his contributions at the early stages of this work. We acknowledge financial support from ERDF: European Regional Development Fund. This research was supported by the Spanish MICINN, through Contract No. FIS2008-01236 and the Generalitat de Catalunya CIRIT, Contract No. 2009SGR-0985.

[1] V. Giovannetti, S. Lloyd, and L. Maccone, Nat. Photonics 5, 222 (2011).

[2] A.S. Holevo, Probabilistic and Statistical Aspects of Quantum Theory, North-Holland Series in Statistics and Probability (North-Holland, Amsterdam, 1982).

[3] C. W. Helstrom, Quantum Detection and Estimation Theory (Academic Press, New York, 1976).

[4] S. Massar and S. Popescu, Phys. Rev. Lett. 74, 1259 (1995).

[5] E. Bagan, M. Baig, A. Brey, R. Muñoz-Tapia, and R. Tarrach, Phys. Rev. Lett. 85, 5230 (2000); Phys. Rev. A 63, 052309 (2001). 
[6] I. D. Ivanovic, Phys. Lett. A 123, 257 (1987).

[7] J. Fiurášek and M. Ježek, Phys. Rev. A 67, 012321 (2003).

[8] H. Sugimoto, T. Hashimoto, M. Horibe, and A. Hayashi, Phys. Rev. A 80, 052322 (2009).

[9] E. Bagan, R. Muñoz-Tapia, G. A. Olivares-Renteria, and J. A. Bergou, Phys. Rev. A 86, 040303 (2012).

[10] S. Massar and S. Popescu, Phys. Rev. A 84, 052106 (2011).

[11] J. Fiurášek, New J. Phys. 8, 192 (2006).

[12] B. Gendra, E. Ronco-Bonvehi, J. Calsamiglia, R. MuñozTapia, and E. Bagan, New J. Phys. 14, 105015 (2012).

[13] B. Gendra, E. Ronco-Bonvehi, J. Calsamiglia, R. MuñozTapia, and E. Bagan (to be published).
[14] See Supplemental Material at http://link.aps.org/ supplemental/10.1103/PhysRevLett.110.100501 for details.

[15] S. Boyd and L. Vandenberghe, Convex Optimization (Cambridge University Press, Cambridge, England, 2004).

[16] E. Bagan, A. Monras, and R. Muñoz-Tapia, Phys. Rev. A 71, 062318 (2005).

[17] This holds in the Bayesian approach followed here. In contrast, in a pointwise approach, where the phase is $a$ priori known to some accuracy, the Heisenberg scaling can be attained already without abstention.

[18] R. Demkowicz-Dobrzański, J. Kołodyński, and M. Guţă, Nat. Commun. 3, 1063 (2012). 